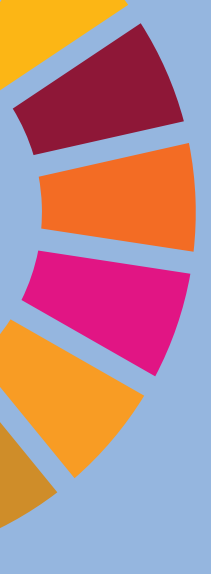

\title{
SALUD DIGITAL: UNA OPORTUNIDAD Y UN IMPERATIVO ÉTICO
}

\author{
Joan Bigorra Llosas \\ Instituto de Salud Global de Barcelona (ISGlobal) \\ Hospital Clínic de Barcelona. \\ joan.bigorra@isglobal.org \\ bigorra@clinic.cat \\ Laura Sampietro-Colom \\ Hospital Clínic de Barcelona \\ Isampiet@clinic.cat
}

\section{RESUMEN}

La salud es imprescindible para conseguir una vida plena, y conseguir una cobertura universal de las prestaciones básicas en salud es una condición esencial para alcanzar no tan sólo el objetivo 3 de los ODS (Objetivos Desarrollo Sostenible) que específicamente habla de salud y bienestar, sino para la totalidad de los 17 objetivos ya que sin salud no hay desarrollo humano ni económico. En los países desarrollados, los sistemas sanitarios están sometidos a una enorme tensión por la inversión de la pirámide demográfica, con el aumento de la esperanza de vida, y por la falta de equidad e ineficiencia en el uso de recursos. En los países en vías de desarrollo la situación es precaria por la enorme carencia de recursos humanos y materiales y la ausencia de infraestructuras. No parece posible, ni quizá deseable, replicar en éstos el modelo sanitario de las economías avanzadas que a pesar de su enorme coste presenta grandes debilidades en términos de equidad, eficiencia y participación de los usuarios. Ante la labor inasumible de universalizar el modelo actual convencional de los sistemas sanitarios, la única opción para alcanzar la cobertura universal es priorizar la salud en las políticas y los presupuestos públicos, dignificar la función de los profesionales sanitarios y potenciar el papel protagonista de la persona como paciente con ayuda de las enormes posibilidades de innovación que ofrecen las nuevas tecnologías y la medicina digital. La digitalización es un instrumento imprescindible para conseguir los objetivos globales de salud y bienestar 2030, pero se requieren liderazgos comprometidos y una transformación radical de nuestra manera de entender la sanidad.

Palabras clave: Innovación y Salud Digital, Sistemas Sanitarios, Cobertura Universal, Equidad y Eficiencia, Liderazgo Transformacional 


\section{ABSTRACT}

Having a satisfactory health status is an absolute must to enjoy a rewarding life; therefore achieving an universal health care coverage is an essential requisite to reach not only the SDG 3 related to Health and Wellness for all by 2030, but to achieve the 17 SGDs, since it is a reality that without health there is neither human nor economic development. In developed countries, Health Care Systems are under huge stress due to the demographic changes, with the increase in life expectancy, and the lack of equity which is becoming an everincreasing problem. In the developing countries, the situation is rather challenging due to the chronic shortness of human and material resources and the lack of infrastructures. In these countries it seems not appropriate or advisable to replicate the health care model of the advanced economies which, in spite of their enormous costs, show severe deficits of equity, efficiency and user involvement and participation. This is why the only way to achieve universal health coverage is to give priority to health in public policies and budgets, to dignify the job of health care professionals and to enhance the prominent role of the citizen as a patient by using the huge innovative possibilities that the new medical technologies and digital medicine bring. Digitalization is an indispensable tool to achieve our global objective of health and wellness for all in 2030, but there is a need for strong and committed leadership and we must radically change the way we approach health care delivery.

Key Words: Innovation and Digital Health, Health Care Systems, Universal Coverage, Equity and Efficiency, Transformational Leadership.

Joan Bigorra es Doctor en Medicina, especialista en Farmacología Clínica y Licenciado en Derecho, Máster en Biomedicina, Biotecnología y Derecho de la Unión Europea. En la actualidad es Director de Innovación del Instituto de salud Global de Barcelona (ISGlobal) y Asesor Senior de Innovación del Hospital Clínic de Barcelona.

Laura Sampietro-Colom es Doctora en Medicina, especialista en Medicina Preventiva y Salud Pública. Executive Máster en Liderazgo y Gestión de la Ciencia y la Innovación. En la actualidad es Directora Adjunta de Innovación y Responsable de la Unidad de Evaluación de la Innovación y las Tecnologías Sanitarias en el Hospital Clínic de Barcelona

\section{INTRODUCCIÓN}

Las tecnologías digitales están ganando rápidamente popularidad en los sistemas de salud y este proceso se ha acelerado en gran medida con la epidemia de COVID19 (Winters et al, 2020). Sin embargo, no existe todavía un consenso generalizado entre los expertos sobre la definición de salud digital. Según la OMS, el término englobaría la telemedicina (prestación de servicios médicos a distancia con apoyo de la tecnología, que va desde simples llamadas telefónicas con profesionales sanitarios hasta complejas operaciones quirúrgicas con robots controlados de forma remota), la teleasistencia o telecuidado (uso de la tecnología para tratar al paciente a distancia desde un punto de vista no sólo médico sino también sociosanitario, en general con un carácter preventivo y de seguimiento), la gestión electrónica de la salud (eHealth) o a través de teléfonos móviles (mHealth), el uso avanzado de ciencias de la computación para la gestión de volúmenes masivos de datos de salud (Big Data), la aplicación de la genómica, la aplicación de la robótica a través de la conexión por internet entre dispositivos (IoT) y la aplicación de la inteligencia artificial (Al) en medicina (WHO, 2019). Aunque existen ya numerosos ejemplos de aplicación de la salud digital tanto en países de renta alta como en países de renta media y baja, hay una relativa falta de estudios sobre los aspectos éticos que esta revolución digital suscita en múltiples ámbitos y puntos de vista. Y por aspectos éticos hay que referirse tanto a los retos que suscita su aplicación como a los que implica su no utilización generalizada y optimizada para aumentar las coberturas sanitarias cuando la tecnología se encuentra disponible y accesible. 


\section{LA SALUD DIGITAL COMO OPORTUNIDAD}

Las herramientas digitales pueden tener efectos muy positivos para la gestión sanitaria y el cuidado de la salud. Sin pretender ser exhaustivos, hay una larga lista de beneficios potenciales asociados a lageneralización de la salud digital y al acceso a datos masivos de salud:

- La digitalización facilita el acceso remoto a la información lo cual permite atender las crecientes necesidades de atención a la salud de las personas con problemas de movilidad o de aquellas poblaciones que viven en zonas remotas o incluso, como ha demostrado la actual pandemia de COVID 19, en otras situaciones donde los desplazamientos y las visitas presenciales no son posibles por razones sanitarias.

- La interacción digital estimula una mayor participación del paciente en el cuidado de su salud. El mayor conocimiento del paciente sobre sus riesgos y su salud a través de herramientas y medidores conectados por medio digitales puede evitar complicaciones, salvar vidas y reducir sufrimientos y costes. Las soluciones digitales permiten por ejemplo controlar los niveles de presión arterial y de glucosa, planificar la nutrición, conectar con los cuidadores, gestionar el cumplimiento terapéutico u organizar las visitas médicas.

- Los recursos digitales podrían compensar la carencia de profesionales de la salud, especialmente en los países de renta más baja y en las zonas de conflicto. Hay que tener en cuenta que en algunos países la ausencia de personal sanitario local es casi total y que nuestros conceptos de centro de salud y hospital de referencia son inexistentes, pero gran parte de la población dispone de dispositivos móviles. Además, es mucho más realista trasladar tecnología médica que esperar a disponer de una masa crítica de profesionales sanitarios debidamente formados. Si se pretende alcanzar algo parecido a la cobertura universal en sanidad en el año 2030 (Objetivo de Desarrollo Sostenible 3), el despliegue rápido de la sanidad digital no es sólo una oportunidad sino también una necesidad ineludible (Wahl B et al, 2018). Algo parecido ocurre en las zonas de conflicto. Es inaceptable que una persona fallezca en una zona de conflicto sin disponibilidad de médicos especialistas por un parto distócico o de una herida perfectamente tratable mediante la cirugía robótica a distancia.

- La digitalización permite potenciar una mejor gestión de las patologías crónicas y mejorar los resultados de salud. Hay estudios piloto muy significativos que demuestran las ventajas incuestionables de esta aproximación. Por ejemplo, en los pacientes con insuficiencia cardíaca congestiva la simple monitorización domiciliaria de la frecuencia cardíaca y la saturación de $\mathrm{O} 2$ con un sensor de bajo coste junto con el seguimiento del peso a través de una balanza con transmisor de señales, permite detectar y corregir muy rápidamente las descompensaciones que de otro modo requerirían atención hospitalaria urgente y pondrían en riesgo la vida del paciente en unos pocos días.

- Contrariamente a lo que pueda parecer, la sanidad digital puede y debe mejorar la relación médico - paciente. De hecho, una mayor digitalización de la sanidad capaz de automatizar sistemas de captación e interpretación de la información sanitaria permitiría a los profesionales dedicar su tiempo a contemplar al paciente en su integridad como persona y a cuidar y curar durante la visita, en lugar de estar atrapado en complejidades tecnológicas o burocráticas. Asimismo, la digitalización puede facilitar una reducción drástica o incluso la eliminación de los errores humanos en el cuidado de la salud.

- La sanidad digital es esencial para mejorar la eficiencia global del sistema sanitario ya que permite una mayor participación y conectividad de todos los actores, mayor acceso a la información y a los sistemas de apoyo a las decisiones clínicas, mayor trasparencia de los resultados de salud ligados a las intervencio- 
nes, así como una importante reducción de los costes de transacción. Cada paciente, cada acto médico es fuente de aprendizaje. No es aceptable que este conocimiento no se aproveche por falta de inversión en digitalización o en formación.

- La digitalización permite potenciar la investigación y la innovación mediante la posibilidad de conocer mejor los mecanismos íntimos de la enfermedad en cada paciente individual a través de la genómica, la proteómica y en general de la biología molecular, pero además la capacidad tecnológica para procesar datos médicos masivos permitiría conocer mejor la epidemiología y la evolución de las enfermedades, así como las posibilidades de prevenirlas y tratarlas de manera eficiente.

- Finalmente la sanidad digital es imprescindible para favorecer una longevidad con calidad de vida, integrando la información genética ( nuestros genes) y epigenética ( el impacto del entorno sobre la expresión de nuestros genes ) para potenciar la longevidad saludable a través de una medicina predictiva, preventiva, personalizada y participada que necesariamente debe complementarse con la conservación y la adecuación del entorno natural, del medio ambiente y de los espacios de socialización urbanos a los que estamos expuestos y que tanto impactan nuestra salud física y mental. Es decir, la llamada Salud Planetaria (Whitmee S, Haines A et al 2015).

\section{ALGUNAS BARRERAS A LA IMPLANTACIÓN DE LA SALUD DIGITAL}

La implantación de la salud digital no está exenta de barreras. La falta de infraestructuras y la heterogeneidad de los sistemas de recogida y almacenamiento de datos es una de las más importantes y que a menudo hace imposible obtener información agregada de calidad ya que los datos sobre pacientes se almacenan en silos. Además, la ausencia de recursos adecuados hace que los mecanismos de aseguramiento de la calidad (por ejemplo, las comprobaciones de la plausibilidad de los datos o la validación de los mismos por parte del clínico o del propio paciente) sean deficitarios. Todo ello puede hacer que el uso de la información digitalizada para alimentar los sistemas de apoyo a la toma de decisiones se vea muy limitada ya que no se puede exponer a los pacientes a riesgos injustificables por falta de calidad de la información (Digital4Care, 2019).

Otra barrera importante es la falta de adaptabilidad de los sistemas que rigen la administración de los sistemas sanitarios y que están pensados para una época pre-digital. En concreto, los sistemas de pago o reembolso de los proveedores sanitarios que suele basarse en pago por actividad o pago por capitación. Ambos tienen serias limitaciones e incluso ofrecen algunos incentivos perversos que pueden repercutir de forma negativa en la calidad o en la eficiencia de la protección de la salud pública y de la asistencia sanitaria. Esto ha motivado una creciente orientación al pago por resultados de salud, aunque en nuestro entorno esta aproximación, que por cierto es el primer paso para evolucionar hacia una correcta financiación de la salud en general y de la salud digital en particular, es todavía muy inicial y se encuentra con muchas resistencias para su implantación. Más allá de los discursos rimbombantes, la realidad es que la resistencia al cambio de los sistemas de salud es desconcertante e injustificable.

Además existen algunas dificultades en los sistemas sanitarios, que no siempre están realmente interesados en la transparencia e interconectividad imprescindibles para conseguir el nivel exigible de eficiencia, o entre los profesionales que en ocasiones y generalmente por falta de información y de análisis completo de las ventajas e inconvenientes, pueden ver amenazado su rol tradicional por la medicina digital, o incluso por parte de los propios pacientes que en algunos casos todavía no son conscientes de que son los auténticos 
protagonistas de un sistema sanitario que necesariamente deberá ser participativo y deberá ponerlos en el centro del sistema. Lo cierto es que no existe un conocimiento suficiente sobre los derechos y obligaciones ni sobre las posibilidades y limitaciones de la salud digital, lo cual genera dudas y confusión.

Otro aspecto crucial es que se requiere un gran esfuerzo para garantizar la seguridad y la confidencialidad absolutas de los datos personales y de salud, al tiempo que debe facilitarse la opción de que los pacientes puedan compartir su información (generalmente anonimizada) de forma libre con otros pacientes, otros profesionales, otros sistemas sanitarios o para proyectos de investigación o innovación que puedan mejorar la atención, el pronóstico, el alcance, la capacidad de resolución o la eficiencia de la prestación de servicios de salud en cualquier escenario. Con todas las garantías éticas y legales, debería mejorar la cultura de compartir, confiar y colaborar entre sistemas sanitarios, profesionales y pacientes, y facilitar el uso masivo de datos para avanzar en la investigación y la innovación médicas a nivel global (Taylor L, 2015, Tseng J et al 2017). Es nuestro derecho como pacientes (¡todos somos pacientes!) dar acceso a nuestra información, en las condiciones de anonimato que decidamos y para los fines que nos parezcan deseables sin encontrarnos resistencias y trabas pretendidamente médicas y burocráticas propias de tiempos ya superados, aunque muchos no lo aprecien, de un mal entendido paternalismo en sanidad. Y es una obligación ética garantizar los derechos a la confidencialidad y respetar la autonomía de la voluntad de los pacientes sobre los posibles usos de los datos sobre su salud.

\section{SALUD DIGITAL, INNOVACIÓN Y OBJETIVOS DE DESARROLLO SOSTENIBLE (ODS)}

La innovación y en particular las estrategias digitales se han aceptado formalmente como un aspecto crítico para alcanzar los Objetivos de Desarrollo Sostenible (Labrique A et al, 2018). La puesta en marcha de servicios de salud eficientes y de elevada calidad es una piedra angular de la agenda global para alcanzar una cobertura universal (WHO 2020). Las intervenciones de salud digital tales como la coordinación entre niveles o nodos asistenciales a través de medios digitales, y los sistemas digitales de apoyo a las decisiones clínicas muestran un potencial considerable para mejorar la calidad y el nivel de integración de la atención sanitaria que reciben los pacientes, pero se requiere un esfuerzo de estandarización muy superior al actual y el compromiso de los gestores y profesionales sanitarios en los diferentes niveles del sistema de salud para un escalado efectivo ( Orton M et al ,2018).

Otro aspecto crucial es el desarrollo y la adopción de soluciones de financiación efectivas de salud digital que permitan al mismo tiempo arquitecturas de información digital coherentes y una agenda de cobertura sanitaria universal. Esto requerirá una intensa cooperación entre emprendedores, desarrolladores, implementadores, decisores políticos y financiadores.

Es fundamental el rol de los emprendedores para cambiar los paradigmas en que están instalados los sistemas sanitarios y esto es válido tanto para los países de renta media o baja como para garantizar el acceso universal a la totalidad de la población en países de renta alta. En una encuesta publicada en la prestigiosa revista New England Journal of Medicine entre médicos hospitalarios y ambulatorios de los EEUU, los encuestados opinaban mayoritariamente que la innovación digital sería liderada por empresas start-up de nueva creación tanto en los hospitales y los sistemas sanitarios (54\%) como en atención primaria (65\%) (Gittlen S, 2017). 


\section{ALGUNOS ASPECTOS ÉTICOS INAPLAZABLES EN SALUD DIGITAL}

En revisiones recientes sobre crisis sanitarias y humanitarias, dos de las principales recomendaciones fueron el imperativo ético de recoger los mejores datos y la necesidad de mejorar los sistemas de información (Blanchett $\mathrm{K}$ et al 2017, Checchi $\mathrm{F}$ et al 2017). Generar los mejores datos implica registrarlos de forma estandarizada (para garantizar comparabilidad), obtenerlos con la metodología adecuada, debidamente protegidos, enfocados a su utilización práctica por parte de los responsables sanitarios y obtenidos a través de un consentimiento (verdaderamente) informado. El concepto de mejores sistemas de información implica garantizar estándares internacionalmente aceptados, así como asegurar la disponibilidad con seguridad y un fácil acceso para la toma de decisiones incluso en situaciones críticas, de manera interconectada y coste - efectiva.

En la reciente crisis humanitaria del Ébola en África Occidental se pusieron claramente de manifiesto las limitaciones de los sistemas basados en papel tanto en el terreno asistencial como en la investigación y el apoyo logístico. El papel es frágil, se rompe con facilidad, es a menudo difícil de leer o de reproducir por no hablar de su inutilidad cuando existen déficits graves de alfabetización o problemas idiomáticos. Además, el papel en sí mismo puede ser un vector de transmisión de enfermedades infecciosas. Por el contrario, las tecnologías digitales han demostrado ser eficaces, incluso en entornos con muy pocos recursos, cuando se implementan correctamente y con la tecnología y el personal con la especialización adecuada (Perakslis ED, 2018).

Por tanto, no parece ético implantar la digitalización en sanidad sin las debidas garantías de confidencialidad y respeto de los derechos fundamentales de las personas, pero a nuestro juicio tampoco es ético demorar la implantación por gestión ineficiente de los recursos económicos, por inercias inaceptables en los sistemas de financiación o por corporativismos injustificables. Ante los retos actuales de los sistemas de salud la inercia no es una opción. Se requiere con urgencia un liderazgo en sanidad que muestre un elevado nivel de exigencia no tan sólo en el análisis ético de las acciones sino también en el análisis ético de las carencias y omisiones. Y en estos momentos no es una cuestión de recursos sino de cualidades esenciales del liderazgo como la empatía, la capacidad de escuchar, la comunicación, la coherencia, la resiliencia, la capacidad de llegar acuerdos, la sensibilidad por el sufrimiento de los demás y la intolerancia con la ineficiencia culpable y las agendas ocultas. 


\section{REFERENCIAS BIBLIOGRÁFICAS}

- Blanchet $\mathrm{K}$ et al. Health in humanitarian crisis 1: evidence on public health interventions in humanitarian crisis. Lancet 2017; (doi.org/10.1016/S0140-6736(16)30768-1)

- Checchi F et al. Health in humanitarian crisis 2: public health information in crisis-affected populations: a review of methodsand their use for advocacy and action. Lancet 2017; (doi.org/10.1016/501406736(17)30702-X)

- Digital4Care. Health First Europe. June 2019. Brussels

- Gittlen S.Can Start-Ups Rescue Health Care? NEJM Catalyst, Insight Report February 23, 2017.

- Labrique A, Vasudevan L, Mehl G, Rooskam E, Hyder AA. Digital health and Health Systems of the Future. Global Health: Science and Practice 2018; 6; Suppl 1:1-2

- Orton M, Agarwal S, Muhoza P, Vasudevan L, Alexander V. Strengthening Delivery of health Services Using Digital Devices. Global Health: Science and Practice 2018; 6; Suppl 1: 61-69

- Perakslis ED. Using digital health to enable ethical health research in conflicto and other humanitarian settings. Conflict and health 2018 (https://doi.org/10.1186/s13031-018-0163-z).

- Taylor L. The ethics of big data as a public good: which public? whose good? Phil Trans A Math Phys Eng Sci. 2015; https//doi.org/10.1098/rsta2016 0126.

- Tseng J, Samag S, FraserD, Landman AB. Catalyzing healthcare transformation with digital health: performance indicators and lessons learned from a digital health innovation groupHealthc. 2017; https://doi.org/10.1016/j.hjdsi.2017.09.003

- Wahl B et al. Artificial Intelligence (AI) and global health: how can Al contribute to health in resource-poor setting ? BMJ Glob Health 2018: 3 e000798, doi: 10.1136/bmjgh-2018-000798

- Winters N, Venkatapuram S, Geniets A, Wynne-Bannister E. Prioritarian Principles for digital heslth in low resource settings. J med Ethics 2020, 46: 259-264

- Whitmee S, Haines A et al. Safeguarding human health in the Anthropocene epoch: report of The Rockefeller Foundation-Lancet Commission on planetary health. The Lancet 2015 (http://dx.doi.org/10.1016/S0140-6736(15)60901-1)

- World Health Organization. WHO guidelines recommendations on digital interventions for health systems strengthening 2019.

- World Health Organization. World Health Statistics 2018. Monitoring Health for the Sustainable Development Goals 2020. 
REVISTA DIECISIETE
a NOTAS Y COLABORACIONES 